\title{
TREES WITH HAMILTONIAN SQUARE
}

\author{
FRANK HARARY AND ALLEN SCHWENK
}

Plummer (see $[2 ;$ p. 69]) conjectured that the square of every block is hamiltonian, and this has just been proved by Fleischner [1]. It was shown by Karaganis [3] that the cube of every connected graph, and hence the cube of every tree, is hamiltonian. Our present object is to characterize those trees whose square is hamiltonian in three equivalent ways.

We follow the terminology and notation of the book [2]. In particular, the following concepts are used in stating our main result. A graph is hamiltonian if it has a cycle containing all its points. The graph with the same points as $G$, in which two points are adjacent if their distance in $G$ is at most 2 , is denoted by $G^{2}$ and is called the square of $G$. The subdivision graph $S(G)$ is formed (Figure 1) by inserting a point of degree two on each line of $G$.
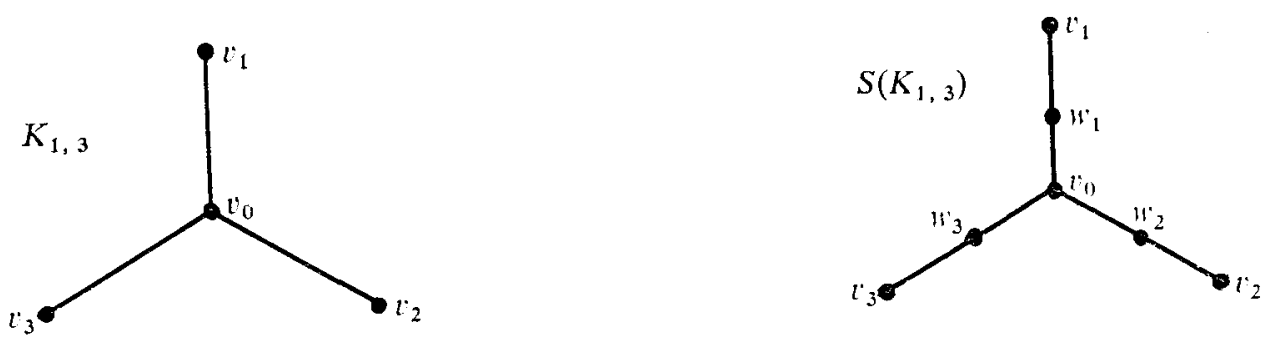

Fig. 1.-The subdivision of the graph $\mathrm{K}_{1,3}$.

The crossing number of $G$, denoted $v(G)$, is the smallest number of crossings of pairs of edges when $G$ is drawn in the plane. We now develop a variation on $v(G)$ for bipartite graphs. To do this, we draw a bigraph $B$ with points of one color on the abscissa and those of the other color at unit distance above the abscissa. We require each line of $B$ to be drawn as a straight line segment. Then the bipartite crossing number $v_{2}(B)$ is the smallest possible number of crossings occurring in all such embeddings of $B$. Figure 2 shows two bigraphs drawn in this way. The first is $S\left(K_{1,3}\right)$ from Figure 1 which has $v_{2}=1$, the second is a tree $T$ with $v_{2}=0$.

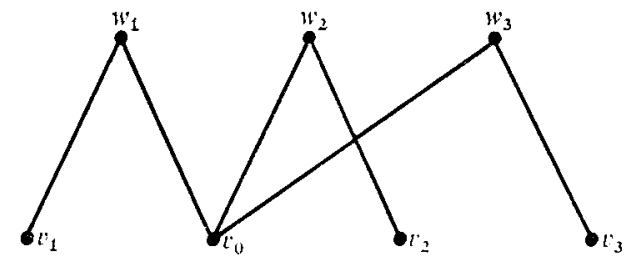

Fig. 2a.

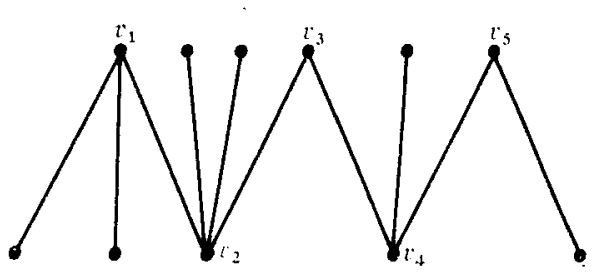

Fig. $2 b$.

Research supported in part by a grant from the Air Force Office of Scientific Research.

[MATHEMATIKA 18 (1971), 138-140] 
We can now state the main theorem which gives several characterizations of trees with hamiltonian square.

THEOREM 1. Let $T$ be a tree at least 3 points. The following statements are equivalent:

(1) $T^{2}$ is hamiltonian.

(2) $T$ does not contain $S\left(K_{1,3}\right)$ as a subgraph.

(3) $T$ minus its endpoints is a path, $P$.

(4) $v_{2}(T)=0$.

Proof. (1) implies (2). Let $C$ be a hamiltonian cycle of $T^{2}$, and assume $T$ has a subgraph $S\left(K_{1,3}\right)$ labelled as in Fig. 1. Let $V_{i}$ be the set of points in the connected component of $w_{i}$ in $T-v_{0}$, for $i=1,2,3$. Certainly $C$ must enter $V_{i}$ and leave it at least once. Since each $\left|V_{i}\right| \geqslant 2$ by the assumption, there must be two distinct points which are the first and last to appear as $C$ passes through $V_{i}$, and one of these, say $u_{i}$, is not $w_{i}$ since $w_{i}$ cannot occur twice in a cycle. Now every path between $T_{i}$ and $T-T_{i}$ must contain $w_{i}$ and $v_{0}$, and so, the only point of $T-T_{i}$ whose distance from $u_{i}$ is at most two is $v_{0}$. But $C$ contains a line between $u_{i}$ and $T-T_{i}$, so this line must be $u_{i} v_{0}$. Since this holds for $i=1,2,3$, it follows that $v_{0}$ is incident with three lines in $C$, a contradiction. Thus, $T$ contains no subgraph $S\left(K_{1,3}\right)$.

(2) implies (3). By hypothesis, $T$ contains no $S\left(K_{1,3}\right)$. Let $T^{\prime}$ be the tree obtained by deleting all the endpoints of $T$. If $T^{\prime}$ is not a path, it has a point of degree at least three. It is then evident that $v$ is the central point of some $S\left(K_{1,3}\right)$ subgraph in $T$. Since this violates the hypothesis, we conclude that $T^{\prime}$ is a path.

(3) implies (4). By hypothesis, $T^{\prime}$ is a path $P$ which can be labelled $v_{1}, v_{2}, \ldots, v_{n}$. Embed $P$ on two parallel lines in a zigzag fashion with no crossing as illustrated in Fig. $2 \mathrm{~b}$ for $n=5$. Then, for each endpoint of $T$ adjacent to $v_{i}$, insert a point on the herizental line opposite $v_{i}$ between $v_{i-1}$ and $v_{i+1}$. The resulting embedding of $T$ has no crossings, so $v_{2}(T)=0$.

(4) implies (1). Suppose $T$ is embedded with no crossings (as in Fig. 2b). It is convenient to label the upper points from left to right by $u_{1}, u_{2}, \ldots, u_{k}$ and the lower points from right to left by $w_{1}, w_{2}, \ldots, w_{p-k}$. Observe that

$$
d\left(u_{i}, u_{i+1}\right)=d\left(w_{j}, w_{j+1}\right)=2,
$$

for $1 \leqslant i \leqslant k-1$ and $1 \leqslant j \leqslant p-k-1$, since any longer path would have to cross itself. Similarly, $d\left(u_{1}, w_{p-k}\right)=d\left(w_{1}, u_{k}\right)=1$. Consequently,

$$
u_{1}, u_{2}, \ldots, u_{k} w_{1}, w_{2}, \ldots, w_{p-k} u_{1} \text {, }
$$

is a hamiltonian cycle in $T^{2}$.

We notice that the hamiltonian cycle found above uses just two lines of $T$, and these are the endlines of a longest path. This happens to be always true.

THEOREM 2. If $T^{2}$ is hamiltonian, then any hamiltonian cycle contains exactly two lines of $T$, and these are the endlines of a longest path. Moreover, the endlines of every longest path lie on some hamiltonian cycle.

Proof. The result is trivial when $T$ has 3 points. We assume the theorem has been proven for trees on fewer than $p$ points, and proceed to prove it for a $p$-point tree $T$, whose square is hamiltonian. 
If $T$ is itself a path, it can be labelled $v_{1}, v_{2}, \ldots, v_{p}$. It is easily seen that $T^{2}$ has a unique hamiltonian cycle, and this cycle contains only $v_{1} v_{2}$ and $v_{p-1} v_{p}$ and no other lines of $T$.

If $T$ is not a path, consider any longest path $P$, and an endpoint $v_{1}$ not lying on $P$. Let $v_{2}$ and $v_{3}$ be the points which precede and follow $v_{1}$ on a hamiltonian cycle $C$ of $T^{2}$. Now either $v_{1}, v_{2}$ and $v_{3}$ are all adjacent in $T$ to a fourth point $v_{4}$ or else $v_{1} v_{2} v_{3}$ is a path in $T$. In either case $d\left(v_{2}, v_{3}\right) \leqslant 2$. Consequently, the graph $\left(T-v_{1}\right)^{2}$ has a hamiltonian cycle $C^{\prime}$ which is formed by deleting $v_{1}$ from $C$. Clearly $P$ is also a longest path of $T-v_{1}$; so by the induction hypothesis, only the endlines of $P$ can appear in the cycle $C^{\prime}$. Consequently, $d\left(v_{2}, v_{3}\right) \neq 1$, so $d\left(v_{2}, v_{3}\right)=2$. Thus, the only lines of $T$ used in $C$ are the end-lines of $P$, completing the proof.

As a consequence of Theorem 2, we notice that if $T^{2}$ is hamiltonian, all its hamiltonian cycles are identical (up to the order of traversing the endpoints adjacent to a common point).

In [4], Neumann developed necessary and sufficient conditions for a given line of a tree $T$ to lie on a hamiltonian cycle, if any, of $T^{2}$. His rather involved result is subsumed by our Theorem 2 .

\section{References}

1. H. Fleischner, " The square of every nonseparable graph is hamiltonian," to appear.

2. F. Hararay, Graph Theory (Addison-Wesley, Reading, Mass. 1969).

3. J. Karaganis, "On the cube of a graph," Canad. Math. Bull., 11 (1968), 295-296.

4. F. Neumann, "On a certain ordering of se vertices of a tree," Casopis Pest. Mat., 89 (1964), 323-339.

University of Michigan.

University of Waterloo.

05C05 Combinatorics: Graph theory; Trees.

05C35 Combinatorics: Graph theory: Paths.

(Received on the 22nd of March, 1971.) 